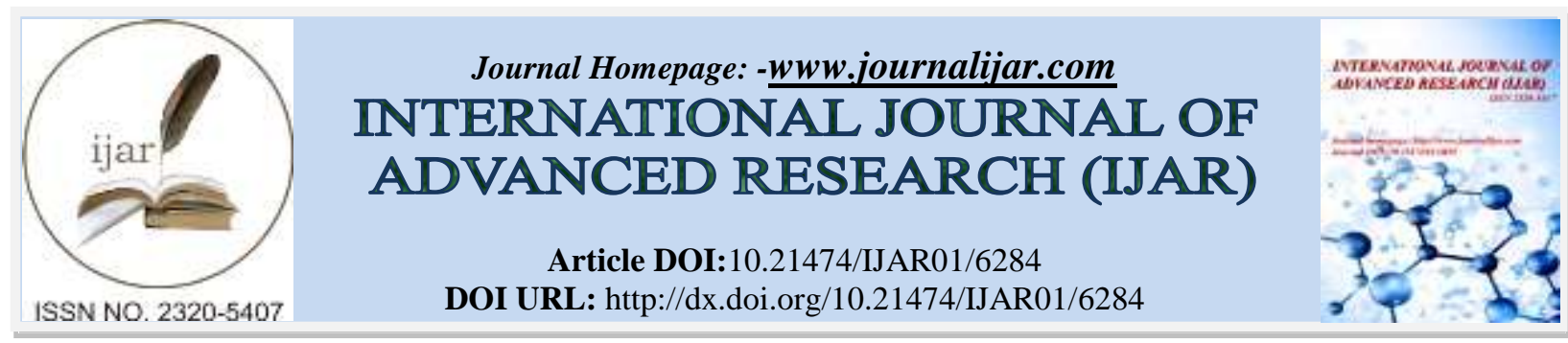

RESEARCH ARTICLE

\title{
CAUSES OF LOW VISION IN VARIOUS OCULAR CONDITIONS.
}

\section{Partha Haradhan Chowdhury ${ }^{1}$ and Brinda Haren Shah ${ }^{2}$.}

1. M.optom, Shree Satchandi Jankalyan Samiti Netraprasikshan Sansthan - Pauri - Affliated to Uttarakhand State Medical Faculty, Dehradun.

2. B.optom, Department of Optometry, Gujarat

\section{Manuscript Info}

Manuscript History

Received: 11 November 2017

Final Accepted: 13 December 2017

Published: January 2018

Key words:-

Low Vision, Causes, Ocular Conditions

\section{Abstract}

This study reveals that why low vision occurs in the specific eye disease. It shows the actual reason for deterioration of vision in case of these diseases. In case of Glaucoma, optic nerve is damaged and due to its anatomical structure peripheral vision is lost and visual acuity is being deteriorated. In case of corneal dystrophy and cataract, improper stimulation comes from the retina to the brain and that's why low vision is being created. In majority of the cases, low vision is being created due to improper stimulation reaches to the part of the brain in various ocular conditions.

Copy Right, IJAR, 2018, . All rights reserved.

\section{Introduction:-}

\section{Glaucoma}

It is also known as optic neuropathy. Here, at first optic nerve head is damaged, it means disc to cup ratio will be changed. It is a progressive disorder. Inspite of lots of another problem in this condition, ultimately whatever the stimulation is needed from retina to the brain will be insufficient.

\section{Corneal Dystrophy}

It is a group of genetic, often progressive bilateral disorder. Usually, it occurs at birth. In this condition, abnormal substances is being deposited at cornea. Critical period is from $1^{\text {st }}$ month of life to $3^{\text {rd }}$ month of life .upto $7^{\text {th }}$ month of life is known as plasticity period. So,in this condition brain growth is progressive too much compared with the rest part of the life. Corneal dystrophy occurs at this time. So, due to improper stimulation from retina to brain, Magnocellular and Parvocellular cells is likely damaged and will create low vision.

\section{Aniridia}

Aniridia means without iris. Usually it is a congenital condition. .Mostly, it is associated with the underdevelopment of macula and optic nerve. It is also associated with nystagmus, glaucoma, cataract and keratopathy. So, in this case, strong light is being encroached to the retina and creates glare problem. It will dazzle the retina. Associated factors can create abnormal stimulation to the brain and hamper magnocellular and parvocellular cells ultimately causing low vision.

\section{Cataract}

Any crystalline lens opacity is called cataract. Here patient will get blurred vision. Congenital cataract develops under critical and plasticity period, so improper stimulation comes from retina to the brain and due to the sensitivity of the brain is high as compared to other time, low vision is created. 


\section{Retinitis Pigmentosa}

It comes under retinal dystrophy. It is a rod cone dystrophy. It is a congenital and progressive condition. With increasing age, this damage is being progressed from the periphery towards the central part of the retina. Macula becomes bony specule and tubular vision is created. Due to this there is no way to get proper stimulation to the brain. That's why, upto certain period of time patient will get low vision and ultimately patient will be total blind.

\section{Retinal Detachment}

It is of two types. Rhegmatogenous and Non- Rhegmatogenous Retinal Detachment. Usually, low vision occurs in cases of Non Rhegmatogenous Retinal Detachment. In this case, no torn will occur. It occurs mainly due to Proliferative Diabetic Retinopathy and Exudative condition. So, fibrovascular tissue is formed at the macular area. Due to improper stimulation at the brain for a prolonged time will create low vision to the patient.

\section{Age Related Macular Degeneration}

It is an age related degenerative condition. Another name is senile macular degeneration. Here, sub retinal neo vascular membrane occur. Main sign is DRUSEN. In this case, images of an object is unable to form at the macular area. So, due to improper stimulation from retina to brain, low vision is created.

\section{Nystagmus}

It is a hereditary and congenital condition. In this case, to and fro eye movement will occur. Generally, it is associated with brain disorder also. In severe cases, improper stimulation to brain occur due to involuntary movement of the eye and the patient will develop low vision.

\section{Retinopathy Of Prematurity}

It occurs in the pre mature babies whose birth weight is less than $1500 \mathrm{gm}$. Here, baby is exposed to the oxygen. Due to this, there is a great chance to develop retinal detachment. This occurs in the critical period, so due to its sensitivity of its timing and due to improper stimulation to the brain will develop low vision

\section{Diabetic Retinopathy}

It mainly occurs due to diabetes mellitus. Diabetic retinopathy is divided into four stages. Non proliferative diabetic retinopathy, clinical significant macular edema, proliferative diabetic retinopathy and advanced proliferative diabetic retinopathy. In the stage of clinically significant macular edema, retina is being thickened $>500 \mu \mathrm{m}$. In the stage of proliferative diabetic retinopathy,neovascularization occurs and will create neo vascularization on disc and neo vascularization elsewhere. So, ultimately fibrovascular tissue is being created on retinal area, so proper stimulation will not be reached at the brain and it will create low vision.

\section{Myopic Degeneration}

In high myopia, this occurs due to long axial length of eyeball and is associated with vitreous liquefaction. There is a chance of developing retinal detachment also. So, associated all of these condition can create blur retinal image and improper stimulation at the brain will create low vision.

\section{Coloboma}

It is defined as incomplete closure of the embryonic fissure of either iris / retina etc. In case of iris, extra light will be encroached onto the retina, retina will become dazzled and glare problem is being created. In case of retinal coloboma, images of an object is not being formed properly, that's why brain will perceive improper stimulation and creates low vision.

\section{Dislocated Lens}

It is defined as complete dislocation of crystalline lens from the pupillary area/ of the eye. Ectopia lentis means partial dislocation. In case of dislocated lens, eye will be high hypermetropic. So, due to improper stimulation into the brain can cause low vision.

\section{Albinism}

It is a group of condition which is associated with eye, skin and hair. In this case, lack of pigmentation and or pigmentary deformity occurs. Albinism is of 3 types. - generalized, cutaneous and oculocutaneous.In cases of oculo 
cutaneous, no pigmentation is present over iris/retina. Then, retina is being showed as a unique appearance due to choroidal showing. Due to absence of pigmentation, light absorption is impossible, so photo sensitivity occurs and associated with severe glare. These patients have underdeveloped fovea so it will create low visual acuity ranging from $6 / 18$ to $6 / 60$ or even lesser. In this case, misrouting of the visual pathway occur and it will create low vision.

\section{References:-}

1. Richard L. Brilliant (1999), Essentials of Low vision practice.

2. Bruce P. Rosenthal, Roy Gordon Cole (1996), Functional Assessment of Low Vision

3. Paul L. Pease (2006) Borish's Clinical Refraction. William J. Benjamin Second ed

4. Randall T. Jose (1983), Understanding low vision

5. Mitchell Scheiman, Maxine Scheiman, Steven Whittaker (2007), Low Vision Rehabilitation: A Practical Guide for Occupational Therapists 